Proceedings

\title{
Shape-Memory Properties of 3D Printed PLA Structures ${ }^{\dagger}$
}

\author{
Guido Ehrmann 1, and Andrea Ehrmann 1,2,* \\ ${ }^{1}$ Virtual Institute of Applied Research on Advanced Materials (VIARAM) \\ ${ }^{2}$ Bielefeld University of Applied Sciences, Faculty of Engineering and Mathematics, Bielefeld, Germany \\ * Correspondence: andrea.ehrmann@fh-bielefeld.de \\ + Presented at the First International Conference on “Green” Polymer Materials 2020, 5-25 November 2020; \\ Available online: https://cgpm2020.sciforum.net/.
}

Published: 4 November 2020

\begin{abstract}
Polylactic acid (PLA) belongs to the few thermoplastic polymers that are derived from renewable resources such as corn starch or sugar cane. PLA is often used in 3D printing by fused deposition modelling (FDM) since it is relatively easy to print, does not show warping and can be printed without a closed building chamber. On the other hand, PLA has interesting mechanical properties which are influenced by the printing parameters and geometries. Here we present shapememory properties of PLA cubes with different infill patterns and percentages. We investigate the material response under defined quasi-static load as well as the possibility to restore the original 3D printed shape. The quasi-static flexural properties are linked to the porosity and the infill structure of the samples under investigation, examined optically and by simulations. Our results underline the importance of designing the infill patterns carefully to develop samples with desired mechanical properties.
\end{abstract}

Keywords: polylactic acid; fused deposition modelling; 3 point bending test; infill parameters; infill density; shape-memory properties

\section{Introduction}

$3 \mathrm{D}$ printing belongs to the emerging technologies of our time. Especially low-cost printers, mostly based on the fused deposition modeling (FDM) technique, are nowadays used by many people, from private people to the maker community to small companies [1]. The mechanical properties of the most often used polymers, such as polylactic acid (PLA) or acrylonitrile butadiene styrene (ABS), and the surface waviness are often the factors limiting possible applications [2-4]. Several research groups thus aim at increasing the mechanical properties, e.g., by embedding nanoparticles, nanofibers, microfibers, chemical or heat treatment [5-7]. Alternatively, PLA or also flexible FDM polymers can be used to create sandwiches with other objects, e.g., with textile fabrics which can be used to increase the elastic modulus [8-10].

Another approach is suggested by some research groups who utilize a special property of PLA, its shape memory [11-13]. Senatov et al. belong to the first who investigated PLA blended with hydroxyapatite to FDM print porous scaffolds which could be strongly mechanically deformed, resulting in cracking of parts of the scaffolds, and afterwards brought back to the original shape in a self-healing process [12]. Liu et al. blended PLA with $\mathrm{SiC}$ and carbon and investigated recovery rate and recovery time of corresponding filaments and printed specimens. They found the first to be correlated with the thermal conductivity, while a higher amount of SiC resulted in higher recovery forces [13]. Wu et al. investigated the impact of diverse printing parameters on the recovery of FDM 
printed PLA samples and found the recovery temperature to be of high importance, with $70{ }^{\circ} \mathrm{C}$ showing optimum results [14].

While many of the aforementioned papers concentrate on blending PLA with different materials or filling the filament with nanoparticles to add a more rigid phase as a backbone, here we use pure PLA and vary the infill pattern of the test samples instead. Similar to the first studies of Senatov et al. $[11,12]$, we perform mechanically destructive tests and investigate the recovery potential.

\section{Experimental}

A 3D printer I3 MK3 (Prusa Research A. S., Prague, Czech) with nozzle diameter $0.4 \mathrm{~mm}$ was used to print the specimens at a layer thickness of $0.15 \mathrm{~mm}$ and a first layer height of $0.2 \mathrm{~mm}$. The nozzle temperature was set to $210^{\circ} \mathrm{C}\left(215^{\circ} \mathrm{C}\right.$ for the first layer), and the bed temperature was $60^{\circ} \mathrm{C}$ during the whole printing process. To examine the impact of the infill patterns alone, no contours were printed so that the whole specimen consisted from 5 compact layers on top and bottom each and pure infill between, with overall dimensions of $20 \mathrm{~mm} \times 20 \mathrm{~mm} \times 20 \mathrm{~mm}$.

As infill patterns, "gyroid" and "3D honeycomb" were chosen, applying $10 \%$ or $15 \%$ infill density. The different specimens are depicted in Figure 1, each from top with a cut above layer 50 as well as from front. Depending on the infill pattern and density, the samples are referred to as $G$ (gyroid) or H (honeycomb), i.e., H10 (Figure 1a), H15 (Figure 1b), G10 (Figure 1c) and G15 (Figure $1 \mathrm{~d})$.

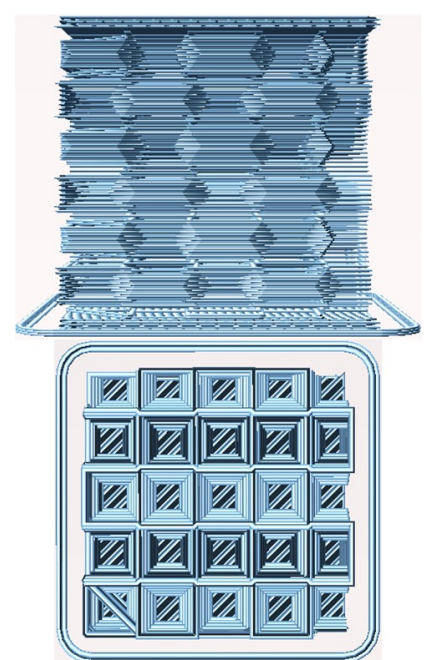

(a)

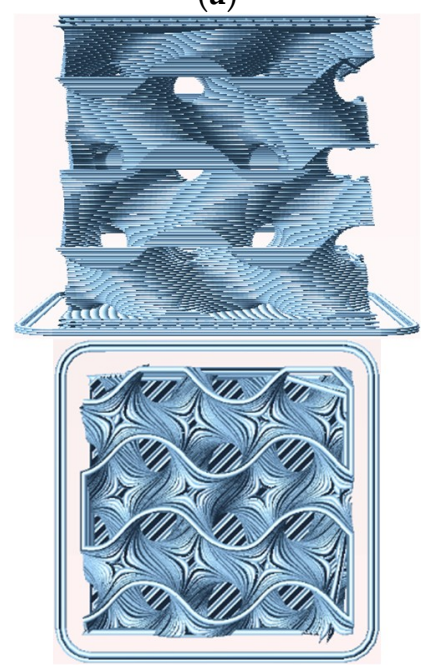

(c)

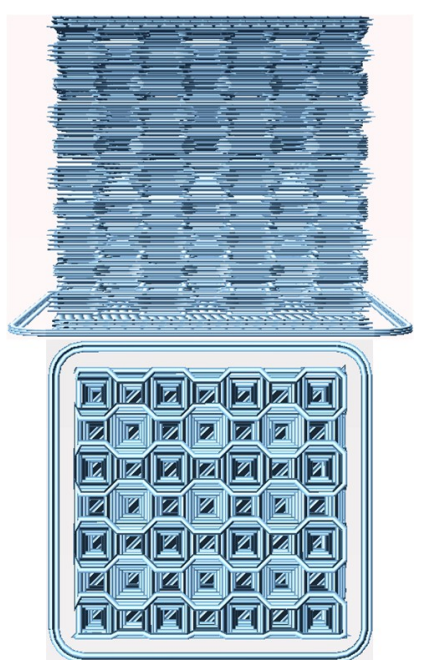

(b)

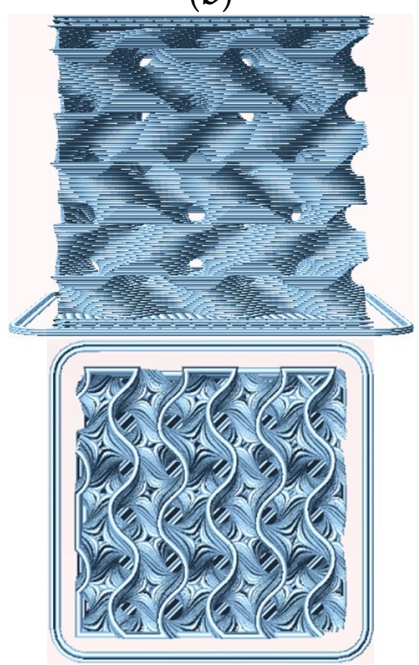

(d)

Figure 1. Front (left) and top view (right) on the samples under examination: (a) 3D honeycomb, 10\% infill; (b) 3D honeycomb, 15\% infill; (c) gyroid, 10\% infill; (d) gyroid, 15\% infill. 
Besides the clearly different wall orientations of the chosen infill structures, Figure 1 also shows that the gyroid structure (Figure 1c,d) has channels crossing the whole specimen, which is not the case for the honeycomb structure.

Investigations of the mechanical properties were performed in a Sauter TVM-N universal testing machine (Kern \& Sohn GmbH, Balingen-Frommern, Germany), combining the single load pin of a 3point bending test with an even counterpart (cf. Figure 2a). In this way, it was possible to test a local impact on the specimen, opposite to previous studies using two flat planes pressing the sample from opposite sides. Quasi-static load tests were stopped at a force of $1700 \mathrm{~N}$ or at a penetration depth of $10 \mathrm{~mm}$, i.e., half the overall sample height. The tests were performed with a speed of $6 \mathrm{~mm} / \mathrm{min}$.

Recovery was accomplished after each test inside a water bath which was held at $(60 \pm 2){ }^{\circ} \mathrm{C}$ for $1 \mathrm{~min}$, directly followed by the subsequent mechanical test. Depending on the visual inspection of the sample after recovery, 5 or 10 test cycles were carried out.

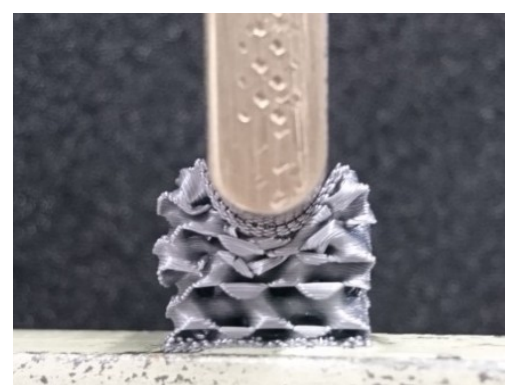

(a)

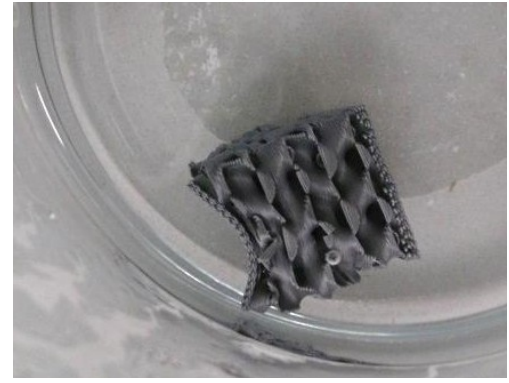

(d)

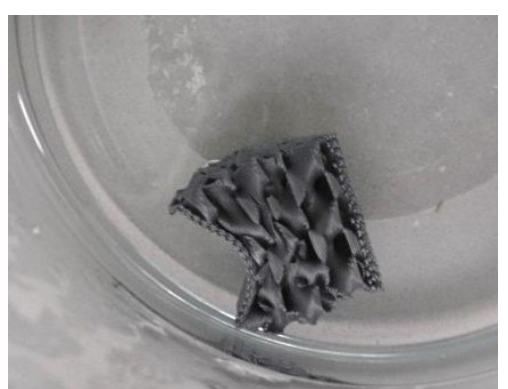

(b)

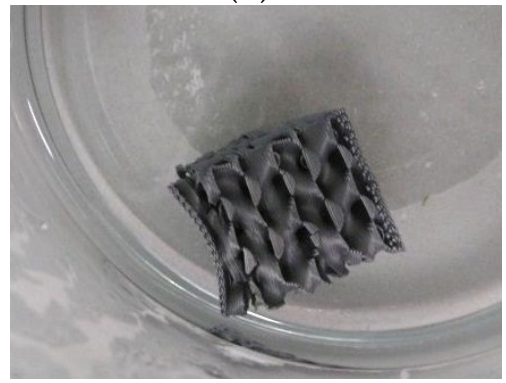

(e)

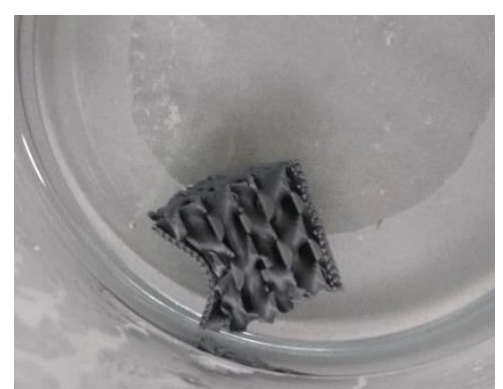

(c)

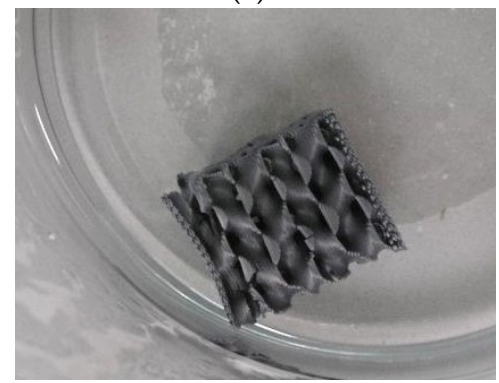

$(\mathbf{f})$

Figure 2. Deformation and recovery processes: (a) Pressing sample G15 up to a maximum impact of $10 \mathrm{~mm}$; (b-f) recovery process in warm water.

\section{Results and Discussion}

Generally, the testing process was performed as depicted in Figure 2, using sample G15 as an example. Firstly, the sample was deformed in the testing machine (Figure 2a). Directly afterwards, the deformed sample was inserted in warm water for recovery (Figure $2 b-f$ ).

This process, however, did not work infinitely long. In each testing cycle, the cracking of single connections which broke under the load was clearly recognizable, indicating that more and more of the sample was destroyed. As an example, Figure 3 depicts sample G15 after 10 deformation cycles and the subsequent-incomplete-recovery which leaves back several clearly visible destroyed bonds and deformations which cannot be restored anymore. 


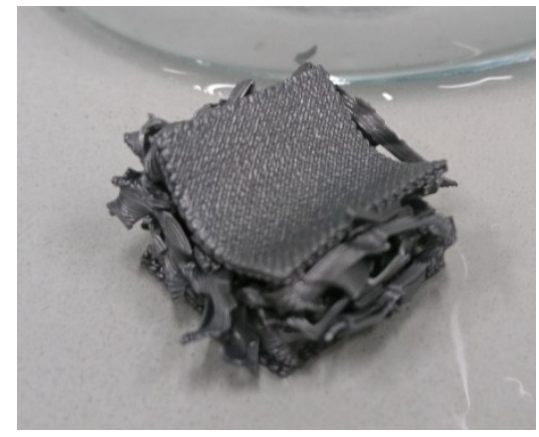

(a)

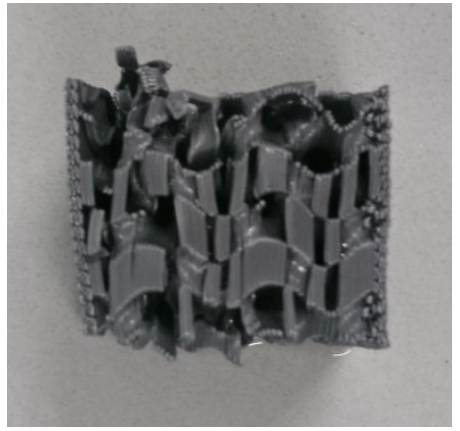

(b)

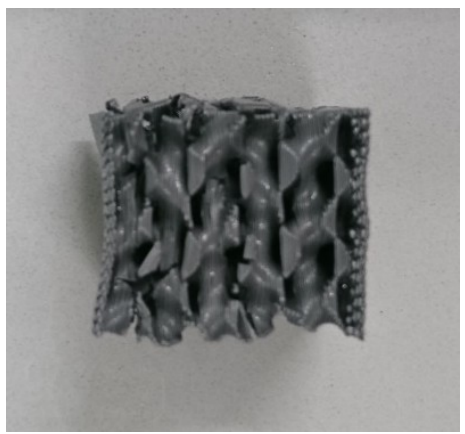

(c)

Figure 3. 10th deformation and recovery processes of sample G15: (a) after maximum deformation; (b-c) after recovery in warm water.

To investigate this behavior quantitatively, quasi-static load tests were performed. The results are depicted in Figure 4. Measurements were stopped after 5 (samples H10, G10) or 10 cycles (samples H15, G15), respectively, depending on the optical appearance, i.e., when clear breaks throughout nearly whole layers became visible.

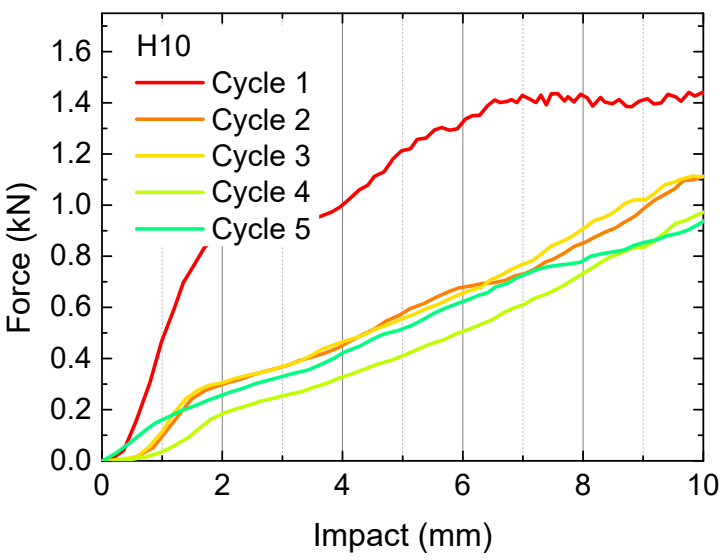

(a)

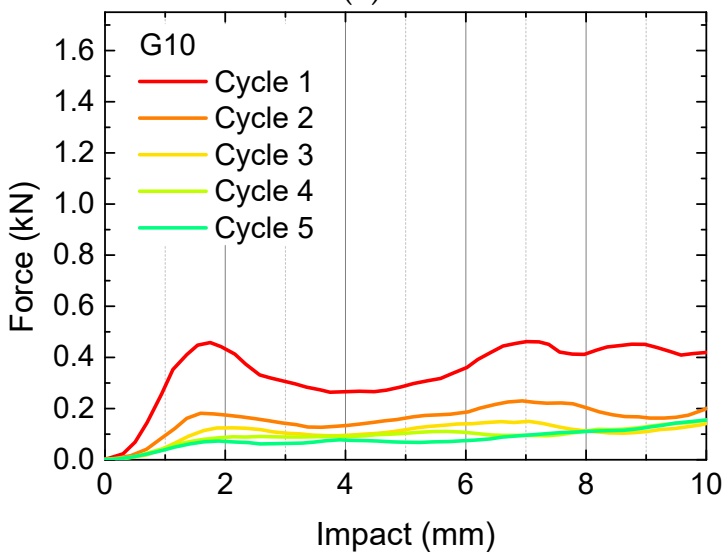

(c)

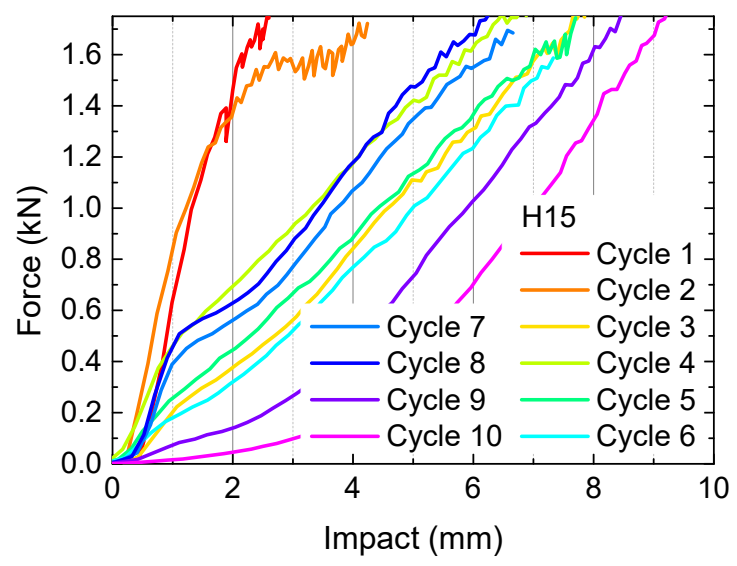

(b)

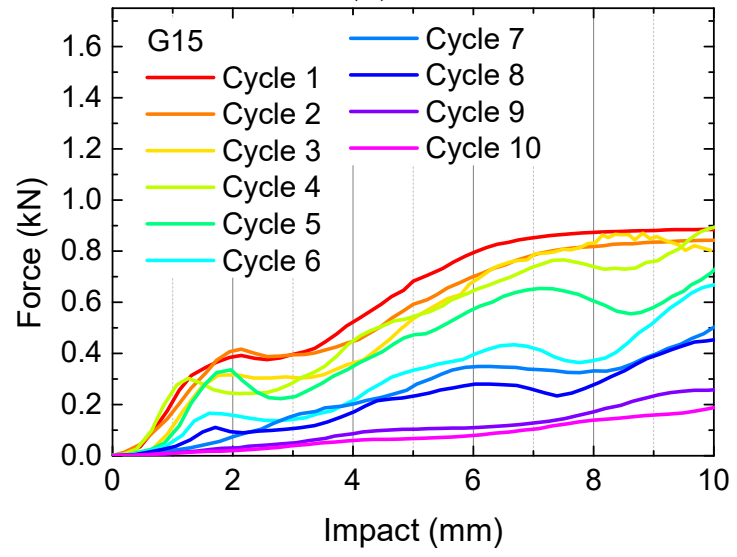

(d)

Figure 4. Quasi-static load tests in original state and after several test and recovery processes: (a) 3D honeycomb, 10\% infill; (b) 3D honeycomb, 15\% infill; (c) gyroid, 10\% infill; (d) gyroid, 15\% infill.

Firstly, Figure 4 clearly shows that the samples with $10 \%$ infill need significantly lower loads for identical impacts than their counterparts with $15 \%$ infill, as expected. Besides, the 3D honeycomb samples show significantly higher loads at identical impact than the gyroid samples. The latter finding can be explained by the structures, depicted in Figure 1. Only the 3D honeycomb samples have vertical walls which can fully counteract the applied forces, while the inner walls in the gyroid 
samples are always tilted, in this way being easier bendable without the necessity to break directly, enabling taking up a certain load by changing the tilting angles of the walls before the material finally breaks at weak points.

What is also visualized here is the fact that recovery is never complete. Under the conditions chosen here, i.e., recovery at $(60 \pm 2){ }^{\circ} \mathrm{C}$ without waiting times before the next test, the forces at identical impact decrease from one cycle to the next in nearly all cases. Deviations can be attributed to small shifts in the position of the sample between subsequent tests. Comparing with Wu et al. [14], next tests should be carried out at a higher temperature of min. $70^{\circ} \mathrm{C}$ to enable possibly a higher amount of recovery.

Another important point must be stated, regarding the 3D honeycomb samples. As mentioned before, this structure does not have channels through which the water in the water bath can flow. This means, on the other hand, that heat transfer in these samples is incomplete, and the inner structures will recover less than the outer ones. Especially for the denser sample H15, this meant that after 10 test cycles, the imprint of the load pin was still visible after recovery, in this way clearly showing that recovery was incomplete.

These first tests of the special infill patterns show that the gyroid filled specimens have a better recovery behavior, but significantly lower load bearing capacities than the 3D honeycomb structure. Next, further tests with recovery at higher temperatures and other structures with channels inside the infill, allowing warm water to penetrate into the whole sample, are necessary to optimize load bearing and recovery properties of FDM printed PLA samples.

\section{Conclusions}

In a recent study, we investigated the recovery properties of porous PLA specimens due to material's shape memory properties. Opposite to previous studies, a 3-point bending load pin was used to apply a local load, and new infill patterns -3D honeycomb and gyroid - were tested. While the first structure showed a significantly higher load bearing capacity, the latter had better recovery abilities.

In general, only a certain amount of recovery could be reached, which may be due to the relatively low recovery temperature of $60{ }^{\circ} \mathrm{C}$, especially in case of the $3 \mathrm{D}$ honeycomb specimens which did not show continuous channels through which the warm water could penetrate into the inner parts of the samples.

Future tests will concentrate on varying the recovery temperature and designing a new structure which combines the advantages of the recent ones.

Author Contributions: G.E. and A.E. designed and performed the experiments and analyzed the data; A.E. wrote the paper. All authors have read and agreed to the published version of the manuscript.

Acknowledgments: This study received no funding.

Conflicts of Interest: The authors declare no conflict of interest.

\section{References}

1. Szykiedans, K.; Credo, W. Mechanical properties of FDM and SLA low-cost 3-D prints. Procedia Eng. 2016, 136, 257-262, doi:10.1016/j.proeng.2016.01.207.

2. Wach, R.A.; Wolszczak, P.; Adamus-Wlodarczyk, A. Enhancement of Mechanical Properties of FDM-PLA Parts via Thermal Annealing. Macromol. Mater. Eng. 2018, 303, 1800169, doi:10.1002/mame.201800169.

3. Fafenrot, S.; Grimmelsmann, N.; Wortmann, M.; Ehrmann, A. Three-Dimensional (3D) Printing of PolymerMetal Hybrid Materials by Fused Deposition Modeling. Materials 2017, 10, 1199, doi:10.3390/ma10101199.

4. Kozior, T.; Mamun, A.; Trabelsi, M.; Sabantina, L.; Ehrmann, A. Quality of the Surface Texture and Mechanical Properties of FDM Printed Samples after Thermal and Chemical Treatment. Stroj. Vestn. 2020, 66, 105-113, doi:10.5545/sv-jme.2019.6322.

5. Ivanov, E.; Kotsilkova, R.; Xia, H.S.; Chen, Y.H.; Donato, R.K.; Donato, K.; Godoy, A.P.; di Maio, R.; Silvestre, C.; Cimmino, S.; et al. PLA/Graphene/MWCNT Composites with Improved Electrical and 
Thermal Properties Suitable for FDM 3D Printing Applications. Appl. Sci. 2019, 9, 1209, doi:10.3390/app9061209.

6. Kumar, R.; Singh, R.; Singh, M.; Kumar, P. ZnO nanoparticle-grafted PLA thermoplastic composites for 3D printing applications: Tuning of thermal, mechanical, morphological and shape memory effect. $J$. Thermoplast. Compos. Mater. 2020, doi:10.1177/0892705720925119.

7. Wickramasinghe, S.; Do, T.; Tran, P. FDM-Based 3D Printing of Polymer and Associated Composite: A Review on Mechanical Properties, Defects and Treatments. Polymers 2020, 12, 1529, doi:10.3390/polym12071529.

8. Grothe, T.; Brockhagen, B.; Storck, J.L. Three-dimensional printing resin on different textile substrates using stereolithography: A proof of concept. J. Eng. Fibers Fabr. 2020, 15, 1558925020933440, doi:10.1177/1558925020933440.

9. Korger, M.; Glogowsky, A.; Sanduloff, S.; Steinem, C.; Huysman, S.; Horn, B.; Ernst, M.; Rabe, M. Testing thermoplastic elastomers selected as flexible three-dimensional printing materials for functional garment and technical textile applications. J. Eng. Fibers Fabr. 2020, 15, 1558925020924599, doi:10.1177/1558925020924599.

10. Kozior, T.; Blachowicz, T.; Ehrmann, A. Adhesion of three-dimensional printing on textile fabrics: Inspiration from and for other research areas. J. Eng. Fibers Fabr. 2020, 15, 1558925020910875, doi:10.1177/1558925020910875.

11. Senatov, F.S.; Zadorozhnyy, M.Y.; Niaza, K.V.; Medvedev, V.V.; Kaloshkin, S.D.; Anisimova, N.Y.; Kiselevskiy, M.V.; Yang, K.-C. Shape memory effect in 3D-printed scaffolds for self-fitting implants. Eur. Polym. J. 2017, 93, 222-231, doi:10.1016/j.eurpolymj.2017.06.011.

12. Senatov, F.S.; Niaza, N.K.; Zadorozhnyy, M.Y.; Maksimkin, A.V.; Kaloshkin, S.D.; Estrin, Y.Z. Mechanical properties and shape memory effect of 3D-printed PLA-based porous scaffolds. J. Mech. Behav. Biomed. Mater. 2016, 57, 139-148, doi:10.1016/j.jmbbm.2015.11.036.

13. Lendlein, A.; Langer, R. Biodegradable, elastic shape-memory polymers for potential biomedical applications. Science 2002, 296, 1673-1676.

14. Wu, W.Z.; Ye, W.L.; Wu, Z.C.; Geng, P.; Wang, Y.L.; Zhao, J. Influence of Layer Thickness, Raster Angle, Deformation Temperature and Recovery Temperature on the Shape-Memory Effect of 3D-Printed Polylactic Acid Samples. Materials 2017, 10, 970, doi:10.3390/ma10080970.

(C) 2020 by the authors; licensee MDPI, Basel, Switzerland. This article is an open access article distributed under the terms and conditions of the Creative Commons by Attribution (CC-BY) license (http://creativecommons.org/licenses/by/4.0/). 